\title{
An Edge Detection Algorithm of Moving Object Based on Background Modeling and Active Contour Model
}

\author{
1Bo Wang, 2Kun Zhang, 3Liang Shi, 4Huihui Zhong \\ 1, First Author Wuhan University of Technology, Hubei, China \\ Qinhuangdao Institute of Technology, Hebei, China, wangb82428@163.com \\ *2,Corresponding Author Northeastern University at Qinhuangdao, Hebei, China, zkhbqhd@163.com \\ 3. Environmental Management College of China, Hebei, China,11996161@qq.com \\ ${ }^{4}$ Qinhuangdao Institute of Technology, Hebei, China, 546736781@qq.com
}

Keywords: background modeling; sparse matrix; active contour model; edge detection.

\begin{abstract}
A novel algorithm based on background modelling and active contour model is proposed for moving object edge detection. Firstly, it uses the background modeling to complete moving object detection, then it uses quad-tree decomposition method to contain the corresponding to the foreground image, through the data distribution density of the sparse matrix, calculates the seed points corresponding to the regions which are containing the moving object. Finally, starting from these seed points, it executes the active contour model in parallel to complete the multiple moving objects edge detection. Experimental results show that the proposed algorithm can effectively obtain the object outlines of multi-moving objects and the edge detection results are close to the judgment of the human visual, parallel contour extraction makes our algorithm has good real-time.
\end{abstract}

\section{Introduction}

With the computer hardware and software technology continues to mature, a variety of multimedia technology begins to flourish, so does the network video monitoring system. In order to deal with the massive recorded video content, it requires an intelligent video surveillance system to analyze the critical object automatically. Currently, main methods of moving object detection are background subtraction [1], frame difference method [2, 3] and optical flow method [4, 5], etc. At present, methods which can be used to light illumination and background slow changes are Kalman filtering and Gaussian mixture model $[6,7]$. On the basis of background modeling, the edge detection of the moving object for further feature extraction and object recognition should be the foundation for intelligent processing. Algorithms based on active contour model [8, 9] are effective methods for edge detection.

This paper proposes a new moving object edge detection algorithm. First, it uses sample statistics of adjacent video frames and Chebyshev inequality to detecting the pixel which consists of the significant background or foreground characters. Secondly, by using the threshold method to complete moving object detection. If there are multiple moving objects in the foreground image, it is also necessary to localization the objects for the moving object detection. Finally, we introduce position seed into active contour model and propose a novel edge detection algorithm based on background modeling and distance model to complete the moving object edge detection effectively. Here for position seeds we use the quad-tree decomposition and sparse matrix block operation for multiple moving objects position detection accurately.

The contents of this paper are organized as follows: in Section 2, we explain the related work of our algorithm, including quad-tree decomposition and active contour model. Section 3 describes the proposed algorithm in detail. Section 4 provides the experimental results and the analysis. Finally, there are conclusion and references.

\section{Related work}


Active contour model is an effective object contour extraction method. Kass [10] proposed active contour model known as the traditional Snake model, which is a semi-interactive algorithms, the basic idea is that: fish set a closed draw in advance, which is close to the true contour curve, then the energy function through the introduction of the internal forces and external forces, making the initial contour changing, eventually converge to the contours of the object.

In traditional Snake model, parametric equation of contour curve $C$ can be expressed as:

$v(s)=[x(s), y(s)], s \in[0,1]$

Wherein: $v(s)$ is the two-dimensional coordinate point on the curve, and $s$ is a normalized arc length. The energy of contour curve $C$ is defined as:

$$
E_{\text {snake }}=\int_{0}^{1}\left[E_{\text {int }}(v(s))+E_{\text {image }}(v(s))+E_{\text {ext }}(v(s))\right] d s=E_{\text {int }}+E_{\text {image }}+E_{\text {ext }}
$$

In the formula (2), $E_{\text {int }}$ is the internal energy, $E_{\text {image }}$ is the energy generated within the image itself, $E_{\text {ext }}$ is the external energy generated by outside restricting force. The internal energy makes control of the smoothness and continuity for the contour curve $C$. The internal energy $E_{\text {int }}$ may be further described as follows:

$$
\begin{aligned}
& E_{\text {int }}(v(s))=\left(\alpha(s)\left|v_{s}(s)\right|^{2}+\beta(s)\left|v_{s s}(s)\right|^{2}\right) / 2 \\
& E_{\text {image }} \text { May be further described as follows: } \\
& E_{\text {image }}=-\left|\nabla\left(G_{\sigma} * I\right)(x(s), y(s))\right|
\end{aligned}
$$

In the formula (4), $\nabla$ is the gradient operator, $G_{\sigma}$ is a two-dimensional Gaussian function with the standard deviation of $\sigma$ and $I$ is the image pixel gray value.

Contour curve $C$ is directed by external energy $E_{\text {ext }}$ towards the object boundary, $E_{\text {ext }}$ can be defined as:

$$
E_{\text {ext }}=-|\nabla I(x, y)|^{2}
$$

Strike a contour is transformed into solving of $E_{\text {snake }}$ minimum value. According to the variational principle, get an Euler-Lagrange equation as follows:

$$
-\alpha(s) \frac{\partial^{2} v}{\partial s^{2}}+\beta(s) \frac{\partial^{4} v}{\partial s^{4}}+\nabla E_{e x t}(v)=0
$$

Traditional Snake model has the following difficulties: very sensitive to the location of the initial contour; for the depth of recessed area, the contour is also difficult to converge to the boundary. Many improved model for the inadequacies of the traditional active contour model have proposed. For example: Cohen [11, 12] proposed balloon force Snake model and distance model. Xu [13] proposed a gradient vector flow (GVF) model which has made gradient information extended farther uniform area through diffusion equation and made the initial contour (away from the boundary of the object) converge to the true boundary of the object. Shen [9] integrated the image global information into the balloon force Snake model, and relaxed the restriction on the position of the initial contour, to some extent overcome the weak boundary leakage disadvantages.

In order to solve the problem of moving object edge detection, we consider using the active contour model to determine the outline of the object. The active contour model convergence results are subject to the initial contour position, so in this paper, the corresponding regional seed of different moving object is introduced into the active contour model, the initial contour of the active contour model is set around the different moving object, convergence of different moving object outline line, and ultimately achieve the purpose of the edge detection of multiple moving objects.

\section{The proposed algorithm}

In this section, we describe the proposed algorithm in detail. The algorithm uses pixel intensity (greyscale or colour) as the basic feature for the background modelling and video moving object detection. Let $X_{1}, X_{2}, \cdots, X_{N}$ be a sample of adjacent $\mathrm{N}$-frame video images, the location of the pixel in 
the image is marked as $(i, j), X_{1}(i, j), X_{2}(i, j), \cdots, X_{N}(i, j)$ represent the pixel gray values in the given position $(i, j)$ for these images. The proposed algorithm is as follows:

(1) Calculate the sample mean $\bar{X}(i, j)$ and sample second-order centre distance $S(i, j)$ for each pixel.

$$
\bar{X}(i, j)=\frac{1}{N} \sum_{k=1}^{N} X_{k}(i, j), S(i, j)=\frac{1}{N} \sum_{k=1}^{N}\left(X_{k}(i, j)-\bar{X}(i, j)\right)^{2}
$$

Here set $\hat{\mu}(i, j)=\bar{X}(i, j)$ and $\hat{\sigma}^{2}(i, j)=S(i, j)$.

(2) Calculate $\sigma_{k}^{2}(i, j)=\left(X_{k}(i, j)-\bar{X}(i, j)\right)^{2}$ and select the right thresholds $T_{1}, T_{2}$ to classify the pixel as background point or foreground point or suspicious point using the following formula:

$$
M_{k}^{1}(i, j)=\left\{\begin{array}{ll}
1 & \left(1-\sigma_{k}^{2}(i, j) / \varepsilon^{2}\right) \geq T_{1} \\
0 & \left(1-\sigma_{k}^{2}(i, j) / \varepsilon^{2}\right) \leq T_{2}
\end{array} \quad \varepsilon=1.414 \hat{\sigma}\right.
$$

When $M_{k}^{1}(i, j)=1$, the pixel is classified as background point. When $M_{k}^{1}(i, j)=0$, the pixel is classified as foreground point. The pixel is classified as suspicious point When $T_{2}<\left(1-\sigma_{k}^{2}(i, j) / \mathcal{E}^{2}\right)<T_{1}$, for suspicious point we use density estimation method for further discrimination.

(4) Calculate $P_{r}\left(X_{k}\right)=\frac{1}{N} \sum_{i=1}^{N} \frac{1}{\sqrt{2 \pi \hat{\sigma}^{2}}} e^{-\frac{1}{2} \frac{\left(X_{k}-X_{i}\right)^{2}}{\hat{\sigma}^{2}}}$ for suspicious point, set threshold $T_{3}$ and classification function:

$$
M_{k}^{2}(i, j)= \begin{cases}1 & P_{r}\left(x_{k}\right) \geq T_{3} \\ 0 & P_{r}\left(x_{k}\right)<T_{3}\end{cases}
$$

When $M_{k}^{2}(i, j)=1$, the suspicious point is identified as a background point. When $M_{k}^{2}(i, j)=0$, the suspicious point is identified as foreground point.

(5) Update the background pixel value using the following formula:

$$
\begin{cases}B_{N}^{1}(i, j)=\sum_{k=1}^{N} X_{k}(i, j) \cdot M_{k}^{1}(i, j), \operatorname{Sum}_{N}^{1}=\sum_{k=1}^{N} M_{k}^{1}(i, j), & (i, j) \notin K \\ B_{N}^{2}(i, j)=\sum_{k=1}^{N} X_{k}(i, j) \cdot M_{k}^{2}(i, j), \operatorname{Sum}_{N}^{2}=\sum_{k=1}^{N} M_{k}^{2}(i, j), & (i, j) \in K \\ B_{N}(i, j)=\frac{B_{N}^{1}(i, j)+B_{N}^{2}(i, j)}{\operatorname{Sum}_{N}^{1}(i, j)+\operatorname{Sum}_{N}^{2}(i, j)}, \quad(i, j) \in K \cup \bar{K} & \end{cases}
$$

Here, $B_{N}(i, j)$ means the pixel value in the position $(i, j)$ of the background image (marked as $B_{N}$ ) for the sample $X_{1}, X_{2}, \cdots, X_{N}, K$ is a collection of suspicious point location.

(6) Complete the foreground image segmentation through background subtraction method. These foreground images are marked as $F_{k}(k=1,2, \cdots, N) . T_{3}, T_{4}$ are empirical values, generally based on the specific test data to select.

$$
F_{k}(i, j)=\left\{\begin{array}{ll}
X_{k}(i, j) & X_{k}(i, j)-B_{N}(i, j) \geq T_{4} \\
255 & X_{k}(i, j)-B_{N}(i, j)<T_{4}
\end{array} \quad(k=1,2, \cdots, N)\right.
$$

(7) Get the corresponding sparse matrix $D_{k}(i, j)$ of the image $F_{k}(i, j)$ through quad-tree decomposition. The sparse matrix $D_{k}(i, j)$ has the same size of the image $F_{k}(i, j)$. The non-zero elements in the sparse matrix is able to describe the size of the area eligible decomposition consistent standard. For example, if $D_{1}(1,1)=16$, it means that in the image $F_{1}(i, j)$, vertices of a rectangular region of pixels $F_{1}(1,1), F_{1}(1,16), F_{1}(16,1)$ and $F_{1}(16,16)$ meet the quad-tree decomposition consistency standards in pixels, i.e. the difference gradation of each pixel in the area does not exceed a certain threshold value, and the size of the region is $16 \times 16$.

(8) Start from the top left corner of the matrix $D_{k}(i, j), m \times m$ being a size standard, the sparse matrix $D_{k}(i, j)$ is divided into a number of small-area block, in each small $m \times m$ block region, calculate the number of element 1 in the block matrix (marked as $N_{m}$ ), and set the threshold value as $T_{\text {seed }}$, if $N_{m}>T_{\text {seed }}$, marking such block matrix as $S^{\omega}\left(\omega=1,2, \cdots, \Omega_{k}\right)$, here $\Omega_{k}$ represents the number of position detection points of the moving object obtained in the foreground image.

(9) For each $S^{\omega}$, calculate the position coordinates of the detection points marked as $\operatorname{seed}^{\omega}\left(\bar{x}_{\omega}, \bar{y}_{\omega}\right)$ : 


$$
\bar{x}_{\omega}=\left\lfloor\sum_{i=1}^{N_{m}} x_{i} / N_{m}\right\rfloor, \bar{y}_{\omega}=\left\lfloor\sum_{i=1}^{N_{m}} y_{i} / N_{m}\right\rfloor
$$

Here $\left(x_{i}, y_{i}\right)$ is the position coordinates of the element 1 in the $S^{\omega},\left(\bar{x}_{\omega}, \bar{y}_{\omega}\right)$ is the average coordinates of all element 1 in the $S^{\omega}$.

(10) For each $\operatorname{seed}^{\omega}\left(\bar{x}_{\omega}, \bar{y}_{\omega}\right)$, calculate the contour curve $C$ can be expressed as:

$\left\{\begin{array}{l}x(s)=\bar{x}_{\omega}+m \cos s \\ y(s)=\bar{y}_{\omega}+m \sin s\end{array} ; v(s)=[x(s), y(s)], s \in[0,1]\right.$

Here we introduce regional seed $\operatorname{seed}^{\omega}\left(\bar{x}_{\omega}, \bar{y}_{\omega}\right)$ into active contour model to complete the initial curve set. As can be seen, the initial curve is set as a circle line which center is $\operatorname{seed}^{\omega}\left(\bar{x}_{\omega}, \bar{y}_{\omega}\right)$ and the radius is $\mathrm{m}$.

(11) Calculate the distance between each pixel and the nearest edge point in the image $F_{k}(i, j)$, and the distance is marked as $d(i, j)$.Define the Potential energy function:

$$
P_{d}(i, j)=-\exp \left[-d(i, j)^{2}\right]
$$

Its potential field is $-\nabla P_{d}(i, j)$, set this potential energy function as a contour external energy, that is $E_{e x t}=P_{d}(x, y)$. External force for the distance model is $F_{e x t}=\nabla E_{e x t}=\nabla P_{d}(x, y)$. It is substituted into equation (6).Using distance potential model, making initial curve converges to the moving object contour, complete the edge detection of moving object.

The proposed algorithm uses Background Modelling, applies quad-tree decomposition to get the corresponding Sparse Matrix of foreground image, and introduces regional seed $\operatorname{seed}^{\omega}\left(\bar{x}_{\omega}, \bar{y}_{\omega}\right)$ into active contour model to complete the initial curve set.and finally takes use of Distance Model for the edge detection of moving objects, so the algorithm is named as BMDM.

\section{Experimental results}

This article uses the Intel(R) Core(TM) 2 CPU 6300@1.86GHz, 1GB memory Personal Computer, in Matlab7.0 platform for experiments. The video images are captured from $336 \times 448$ traffic surveillance video. Generally we get the adjacent 20 to 30 frames for experiment. Three frame images for examples are shown in Fig 1.

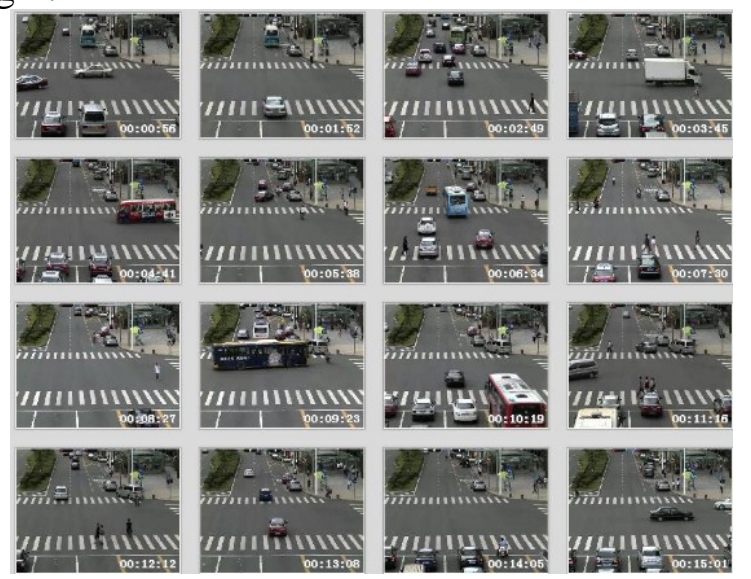

Fig 1. Surveillance video images

Background modeling and foreground image extracting experimental results are shown in Fig 2 and Fig 3. The value of $\varepsilon$ changes between $\hat{\sigma}$ and 2, in the experiment, we set the threshold of $T_{1}$ changes between 0.5and 0.7, $T_{2}$ changes between 0.1 and 0.4. Different experimental results corresponding to threshold $N$ and $T_{1}, T_{2} T_{3}$ different settings are shown in Fig 2. Compare Fig 2-(a) and Fig-2-(d)), it can be seen that the background image can be timely updated. If the state of the object is change (from the rest state to the moving state) the background modeling algorithm can effectively deal with this situation. It has a good real-time. 


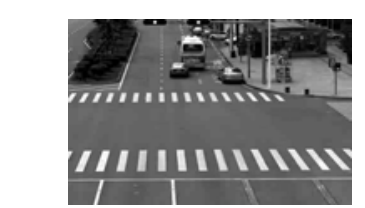

(a) $\begin{aligned} & N=20, T_{1}=0.613 \\ & T_{2}=0.151, T_{3}=0.01\end{aligned}$

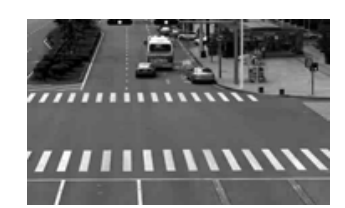

(b) $N=20, T_{1}=0.613$,

(b) $T_{2}=0.151, T_{3}=0.005$

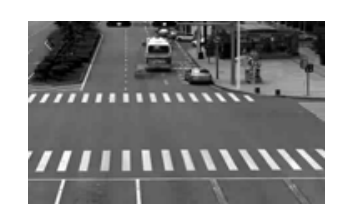

(c) $N=40, T_{1}=0.613$,

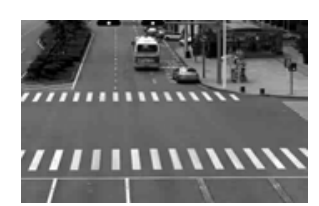

(d) $N=80, T_{1}=0.613$,

(d) $T_{2}=0.151, T_{3}=0.01$

Fig 2. Background modeling experimental results

According to the different real-time background, foreground image can be obtained correspondingly, as shown in Fig3. These foreground images contains more moving objects.

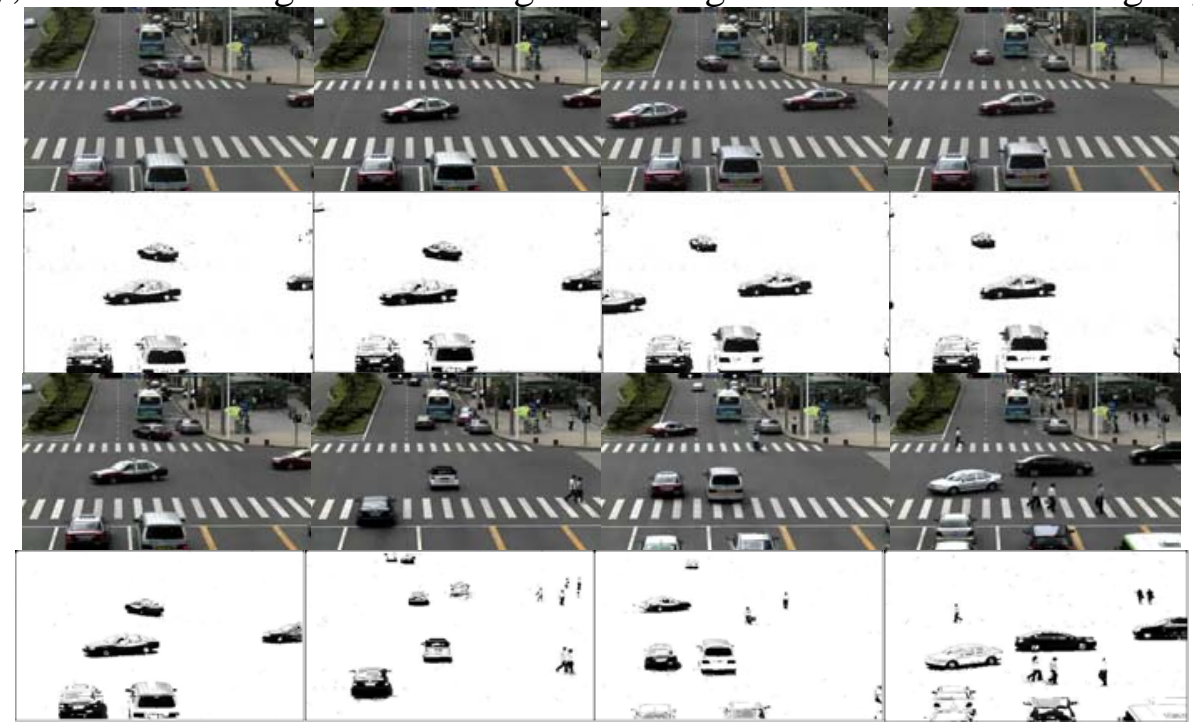

Fig 3. Fore-ground image extraction

The proposed algorithm can get satisfactory moving object segmentation image, the experimental results of quad-tree decomposition for those foreground images are shown in Fig 4-(a), (b), (c), (d). Set $m=32, T_{\text {seed }}=32$ and do the sparse matrix block statistics, obtain the position coordinates corresponding to the different moving object. The experimental results of image-1 are shown in Fig 4-(e), (f), (g), (h).
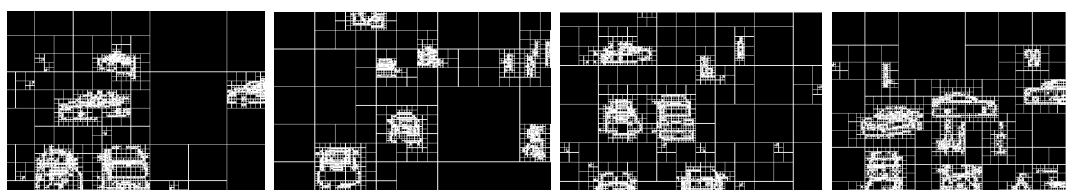

(a) Image -1

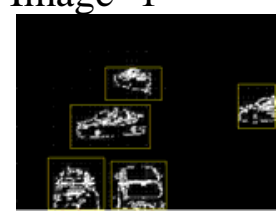

(b) Image -2

(c) Image -3
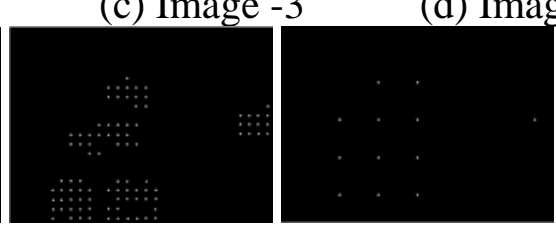

(e) Position detection

(f) $m=32, T_{\text {seed }}=32$

(g) $m=16, T_{\text {seed }}=12$

(h) $m=64, T_{\text {seed }}=55$

Fig 4. Quad-tree decomposition and position detection

As can be seen from Fig4- (f), (g), (h), sparse matrix block size $m \times m$ and the threshold value $T_{\text {seed }}$ can determine the density of the moving object position calibration. These two values should be set related to the size and the number of the moving objects in the foreground image. More moving object, the value of $m \times m$ should be smaller, while adjusting the threshold value $T_{\text {sed }}$ which should be smaller than the value of $\mathrm{m}$. The corresponding regional seed of different moving object is introduced into the active contour model. The initial contour of the active contour model is set around the different moving object, convergence of different moving object outline line. Based on the distance model, moving object edge detection experimental results are shown in Fig 5. 


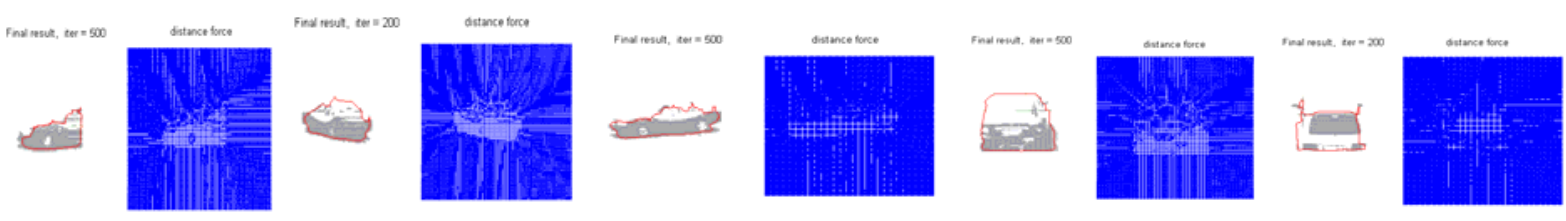

Fig 5. Multi-object edge detection of BMDM algorithm

In order to test the effect of BMDM algorithm for edge detection,we choice the traditional snake model, balloon force snake model and GVF-Snake model as the comparison algorithm. Different algorithms experimental results are shown in Fig 6.
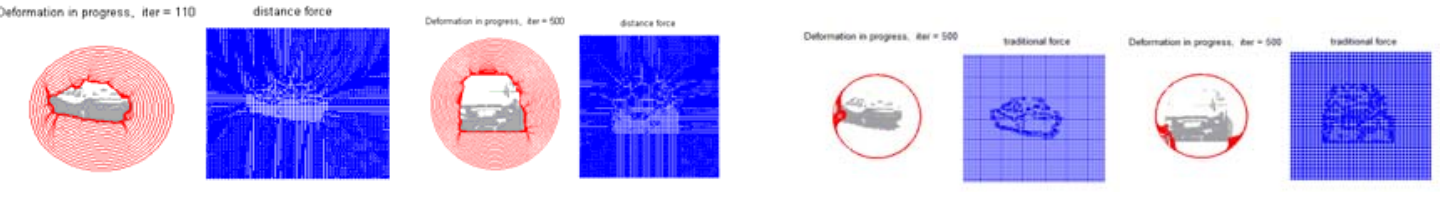

(a) Edge detection based on BMDM algorithm (b) Edge detection based on traditional snake model,
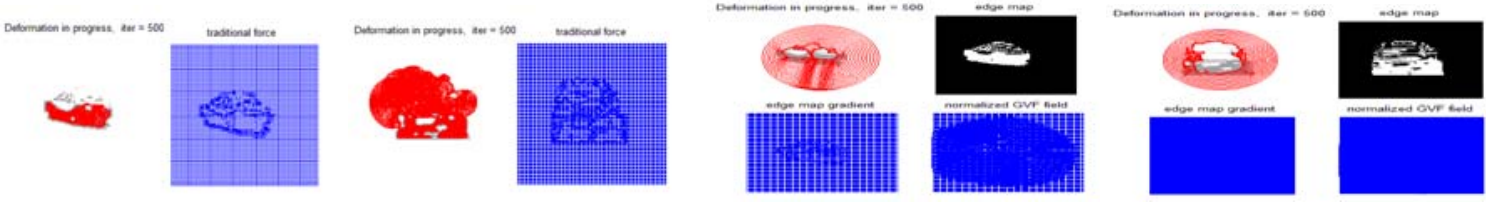

(c) Edge detection based on balloon force snake model(d) Edge detection based on GVF-Snake model

Fig 6. Comparison of different algorithms edge detection

From Fig 6, it can be seen that the proposed BMDM algorithm using distance model can get ideal boundary curve, Edge detection is better than the traditional snake model, balloon force snake model and GVF-Snake model. The traditional snake model can not converge to severe depression boundary, balloon force snake model has boundary overflow, and the boundary got through GVF-Snake model vary greatly from the true. BMDM algorithm(regional seed-based) makes parallel multi-objective edge detection, and it has strong real-time to adapt to the monitoring system.

\section{Conclusion}

In this paper, relating to the moving object edge detection we proposed the BMDM algorithm. The BMDM algorithm uses background modelling, and applies quad-tree decomposition to get the corresponding sparse matrix of foreground image, and finally takes use of distance model for the moving object edge detection. First we calculate the characteristic values (the sample mean and sample variance) for each pixel of the sequence of video frames, then we set and adjust the thresholds $T_{1}, T_{2} T_{3}$ based on experimental data, through background modeling, obtain the foreground images. Finally through sparse matrix block computing get the multiple moving objects position seeds, we introduce these location seeds into distance model, and complete the moving object edge detection. The experimental results show that the moving object edge detection results of the BMDM algorithm are significantly better than the traditional snake model, balloon force snake model and GVF-Snake model. The ideal edge detecting result makes the BMDM algorithm is more suitable for intelligent video surveillance system.

\section{References}

[1] S. Messelodi, C. M. Modena, N. Sehata. A kalman filter based background updating algorithm robust to sharp illumination changes [C]. ICIAP 2005. Italy, (2005) 163-170

[2] J. Badenas, M. Bober, F. Pla. Segmenting traffic scenes from grey level and motion information [J], Pattern Analysis and Applications, (2001)4(1):28-38.

[3] B. Csaba, S. David. Multiple object tracking by hierarchical association of spatio - temporal data, 17th IEEE International Conference on Image Processing, (2010) 41-44. 
[4] R. B. Carlos, J. Femando and G. Nareiso. Visual tracking of multiple interacting objects through Rao-Blackwellized Data Association Particle Filtering [C], $17^{\text {th }}$ IEEE International Conference on Image Processing, Hong Kong, (2010) 821-824

[5] Z. Shuifa, Z. Wensheng, D. Huan and Y. Liu, "Background modeling and object detecting based on optical flow velocity field,” Journal of Image and Graphics, 2nd ed., vol.16, (2011) 236-243.

[6] G. Jean, K. Akio, C. K. Avinash, “ A multi-Kalman filtering approach for video Tracking of human-delineated objects in cluttered environments,” Computer Vision and Image Understanding, 3rd ed., vol.102 (2006) 260-316,

[7] D. Lee. Effective Gaussian mixture learning for video background subtraction [J]. IEEE Trans. On Pattern Analysis and Machine Intelligence, vol.27 (2005) 827-832.

[8] F. Yuqiang, D. Bin, S. Jinze, SHAN Enzhong. An improved moving objects segmentation method based on optical flow technique and active contour model [J]. Journal of Central South University (Science and Technology), (2011) 42(4):1035-1042.

[9] S. Xuan-jing, W. Kaiye, Q. Qingji, L. Yingjie, L. Xiang. Image segmentation using adaptive balloon force snake model[J]. Journal o f Jilin University ( Engineering and Technology Edition), (2011) 41(5):1394-1400.

[10] Kass M, Witkin A, Terzopolous D. Snakes: active contour models [J]. International Journal of Computer Vision, 1988, 1( 4):321- 331.

[11] Cohen L D. On active contour models and balloons [J]. CVGIP: Image Understanding, 1991, 53 (2):211-218.

[12] Cohen L D, Cohen I. Finite- element methods for active contour models and balloons for 2-D and 3-D images [J]. IEEE Transactions on Pattern Analysis and Machine Intelligence, 1993, 15(11):1131-1147.

[13] Xu C, Prince J L. Snakes, shapes, and gradient vector flow [J]. IEEE Transactions on Image Processing, 1998, 7(3):359-369. 